Original article

\title{
INFLUENCE OF EXPERIMENTALLY INDUCED EIMERIA TENELLA INFECTION ON GENE EXPRESSION OF SOME HOST RESPONSE FACTORS IN CHICKENS
}

\author{
A. MILANOVA ${ }^{1}$, R. R. SANTOS ${ }^{2}$, L. LASHEV ${ }^{1}$, V. KOINARSKI ${ }^{3}$ \\ \& J. FINK-GREMMELS ${ }^{2}$ \\ ${ }^{1}$ Department of Pharmacology, Physiology of Animals and Physiological Chemistry, \\ ${ }^{3}$ Department of Veterinary Microbiology, Infectious and Parasitic Diseases, Faculty of \\ Veterinary Medicine, Trakia University, Stara Zagora, Bulgaria; ${ }^{2}$ Division of Veterinary \\ Pharmacology, Pharmacotherapy and Toxicology, Institute for Risk Assessment Sciences, \\ Faculty of Veterinary Medicine, Utrecht University, Yalelaan Utrecht, the Netherlands
}

\section{Summary}

Milanova, A., R. R. Santos, L. Lashev, V. Koinarski \& J. Fink-Gremmels, 2016. Influence of experimentally induced Eimeria tenella infection on gene expression of some host response factors in chickens. Bulg. J. Vet. Med., 19, No 1, 47-56.

Coccidiosis in poultry is associated with significant damage of intestinal integrity and involvement of innate immunity. Therefore, the aim of this study was to evaluate genes, encoding the innate immune response in broiler chickens, such as LEAP, IFN- $\gamma$, iNOS and IL-8 together with PepT1 and ABCG2 transporters after $E$. tenella challenge. E. tenella infection provoked up-regulation of iNOS mRNA in the duodenum and of IFN- $\gamma$ and iNOS mRNAs in the caecum and the liver. IL-8 mRNA expression in the liver was increased and LEAP mRNA was decreased. On the sixth day after the challenge with E. tenella, ABCG2 mRNA was increased in the duodenum and the liver while PepT1 mRNA was upregulated in the duodenum. The expression of these genes in the caecum was not changed. In conclusion, several factors of the host immune response can be affected by E. tenella infection not only in the target caecal tissue but also in the liver and to a lesser degree in the duodenum. The caecal coccidiosis can provoke significant changes in the integrity of the intestines by affecting of ABCG2 and PepT1 mRNA levels.

Key words: chicken, Eimeria tenella, host response, mRNA expression levels

\section{INTRODUCTION}

Coccidiosis in poultry associated with Eimeria spp. infection is a common disease. The implementation of effective control strategies requires understanding of the host response to this infection.
After first invasion of Eimeria spp., innate immunity plays an important role. Previous investigations demonstrated the significance of the Toll-like receptors (Zhang et al., 2012), the role of pro-inflammatory 
cytokines as well as balance of $\mathrm{T}$-helper 1/T-helper 2 (Th-1/Th-2) in the host response to this protozoan infection (Laurent et al., 2001). In particular, the early expression of Th1 cytokines seems important for protection from severe clinical signs of disease (Dalloul \& Lillehoj, 2006).

In addition to markers of the host immune system, antimicrobial peptides have been identified as key mediators of the innate immune system, providing the first line of defense against potential pathogens (Michailidis, 2010). Recently, the participation of antimicrobial peptides such as liver expressed antimicrobial peptide (LEAP) in the innate immune response against Eimeria spp. was studied by Casterlow et al. (2011) and it has been found that its expression was negatively correlated with the severity of coccidian infection. Therefore, LEAP expression may serve as a predictor of the host response to Eimeria infections.

Coccidiosis is accompanied with loss of appetite which results in lower body mass and impaired feed conversion ratio. Nutrient utilisation in chickens depends on the function of transporter proteins in the intestines. One of the key transporters is peptide transporter 1 (PepT1) involved in absorption of di- and tripeptides (Madsen \& Wong, 2011). The expression of PepT1 can be influenced by numerous factors such as food deprivation, pathological conditions and drug administration (Rubio-Aliaga \& Daniel, 2008; Hu et al., 2010). So far there are no published investigations on the effect of coccidiosis on the PepT1 expression or function. ABCG2 and its protein breast cancer resistance protein (BCRP), an $\mathrm{ABC}$ efflux transporter, are expressed at the apical brush-border membrane of enterocytes and participate in the first line defense against xenobiotics, including toxins (van der Heijden et al., 2004; Dahan \& Amidon, 2009). However, the effect of coccidiosis and sulfachlorpyrazine treatment on the level of expression of ABCG2 has not yet been investigated.

The aim of this study was to evaluate the mRNA expression level of selected genes, encoding components of the innate immune response, such as LEAP, interferon gamma (IFN- $\gamma$ ), inducible nitric oxide synthase (iNOS) and interleukin-8 (IL-8) after E. tenella challenge. Attention was paid also to PepT1 and ABCG2 transporters which are involved in the uptake of nutrients and in the efflux of xenobiotics, including drugs used to control poultry diseases.

\section{MATERIALS AND METHODS}

\section{Animals}

The experiments were performed with broiler chickens $(n=14)$ from both sexes (White Plymouth Rock $\times$ Cornish). One day old animals were obtained from a commercial poultry farm and were reared in cages, on a slat floor and under conditions excluding an additional invasion with Eimeria spp. Chickens had free access to standard commercial feed (without additives) and to water and were kept at suitable temperature according to the age requirements of this species. The birds were examined to confirm that they were free of coccidiosis and infectious diseases before the experimental infection with $E$. tenella. The experiments were performed after approval from the Ethical committee of Faculty of Veterinary Medicine, Trakia University.

\section{Experimental infection}

Experimental animals $(n=7)$ were infected at the age of 15 days with an Eimeria 
Table 1. Specific gene primers used in the study

\begin{tabular}{|c|c|c|c|}
\hline Gene & $\begin{array}{l}\text { NCBI accession } \\
\text { number }\end{array}$ & Primers & $\mathrm{Ta},{ }^{\circ} \mathrm{C}$ \\
\hline ABCG2 & NM_001257386.1 & $\begin{array}{l}\text { Forward 5' } \rightarrow \text { 3': CCTACTTCCTGGCCTTGATGT } \\
\text { Reverse 5' } \rightarrow 3^{\prime}: \text { TCGGCCTGCTAAGCTTG }\end{array}$ & 62 \\
\hline IFN- $\gamma$ & NM_205149 & $\begin{array}{l}\text { Forward 5' } \rightarrow 3^{\prime}: \text { CAAGCTCCCGATGAACGAC } \\
\text { Reverse } 5^{\prime} \rightarrow 3^{\prime}: \text { GCAATTGCATCTCCTCTGAGAC }\end{array}$ & 64 \\
\hline Il-8 & NM_205018 & $\begin{array}{l}\text { Forward 5' } \rightarrow 3^{\prime}: \text { CACGTTCAGCGATTGAACTC } \\
\text { Reverse 5' } \rightarrow 3^{\prime}: \text { GACTTCCACATTCTTGCAGTG }\end{array}$ & 64 \\
\hline iNOS & U46504.1 & $\begin{array}{l}\text { Forward } 5^{\prime} \rightarrow 3^{\prime}: \text { GGACAAGGGCCATTGCACCA } \\
\text { Reverse } 5^{\prime} \rightarrow 3^{\prime}: \text { TCCATCAGCGCTGCGCACAA }\end{array}$ & 61 \\
\hline LEAP & NM_001001606.1 & $\begin{array}{l}\text { Forward 5' } \rightarrow 3^{\prime}: \text { CTCAGCCAGGTGTACTGTGCTT } \\
\text { Reverse 5' } \rightarrow 3^{\prime}: \text { CGTCATCCGCTTCAGTCTCA }\end{array}$ & 65 \\
\hline PepT1 & AY029615.1 & $\begin{array}{l}\text { Forward 5' } \rightarrow 3^{\prime}: \text { CCCCTGAGGAGGATCACTGTT } \\
\text { Reverse 5' } \rightarrow 3^{\prime}: \text { CAAAAGAGCAGCAGCAACGA }\end{array}$ & 58.7 \\
\hline$\beta$-Actin & NM_205518 & $\begin{array}{l}\text { Forward } 5^{\prime} \rightarrow 3^{\prime}: \text { ATGTGGATCAGCAAGCAGGAGTA } \\
\text { Reverse } 5^{\prime} \rightarrow 3^{\prime}: \text { TTTATGCGCATTTATGGGTTTTGT }\end{array}$ & 61 \\
\hline
\end{tabular}

Breast cancer resistance protein 2 (ABCG2), interferon gamma (IFN- $\gamma$ ); interleukin (IL-8); inducible nitric oxide synthase (iNOS); liver expressed antibiotic peptide (LEAP), peptide transporter 1 (PepT1); $\mathrm{NCBI}$ - the National Centre for Biotechnology Information; Ta - optimal annealing temperature.

tenella strain isolated from naturally invaded chickens. The strain was enriched via transmission through susceptible birds. Each chicken was infected with $0.4 \mathrm{~mL}$ volume of $E$. tenella suspension, containing $8 \times 10^{4}$ sporulated oocysts, by instillation into the crop. Clinical signs of infection were seen in most animals within 6 days of inoculation.

Study design

The chickens from infected group were euthanised by cervical dislocation by the $6^{\text {th }}$ day after the challenge with E. tenella. Tissue samples (duodenum, caecum and liver) for parasitological and qRT-PCR analysis were taken. Tissue samples were collected at the same time from seven uninfected broilers that served as healthy controls. Samples for qRT-PCR were snap frozen in liquid nitrogen and stored at $-70{ }^{\circ} \mathrm{C}$ until analysis.

BJVM, 19, No 1
$R N A$ isolation, reverse transcription and quantitative real-time polymerase chain reaction analysis

Total RNA was isolated using Qiagen column method (RNeasy Mini Kit, Qiagen, Cat. No 74104). RNA concentrations were determined by ultraviolet absorbance at 260 and $280 \mathrm{~nm}$, and were stored for a short period at $-70{ }^{\circ} \mathrm{C}$. Singlestranded cDNAs were synthesised from 4 $\mu \mathrm{g}$ total RNA using the First Strand cDNA Synthesis Kit (Fermentas Life Science) on a Quanta Biotech QB-96 (Quanta Biotech Ltd.). The reaction mixture (total volume $20 \mathrm{~mL}$ ) was incubated according to the manufacturer's instructions: for $60 \mathrm{~min}$ at $37{ }^{\circ} \mathrm{C}$, and then the enzyme was heat inactivated at $70{ }^{\circ} \mathrm{C}$ for 5 min and the reaction mixture rapidly cooled to $4{ }^{\circ} \mathrm{C}$.

Specific primers for ABCG2, IFN- $\gamma$, IL-8, PepT1, LEAP and iNOS and $\beta$-actin were used (Table 1). Sybr Green 


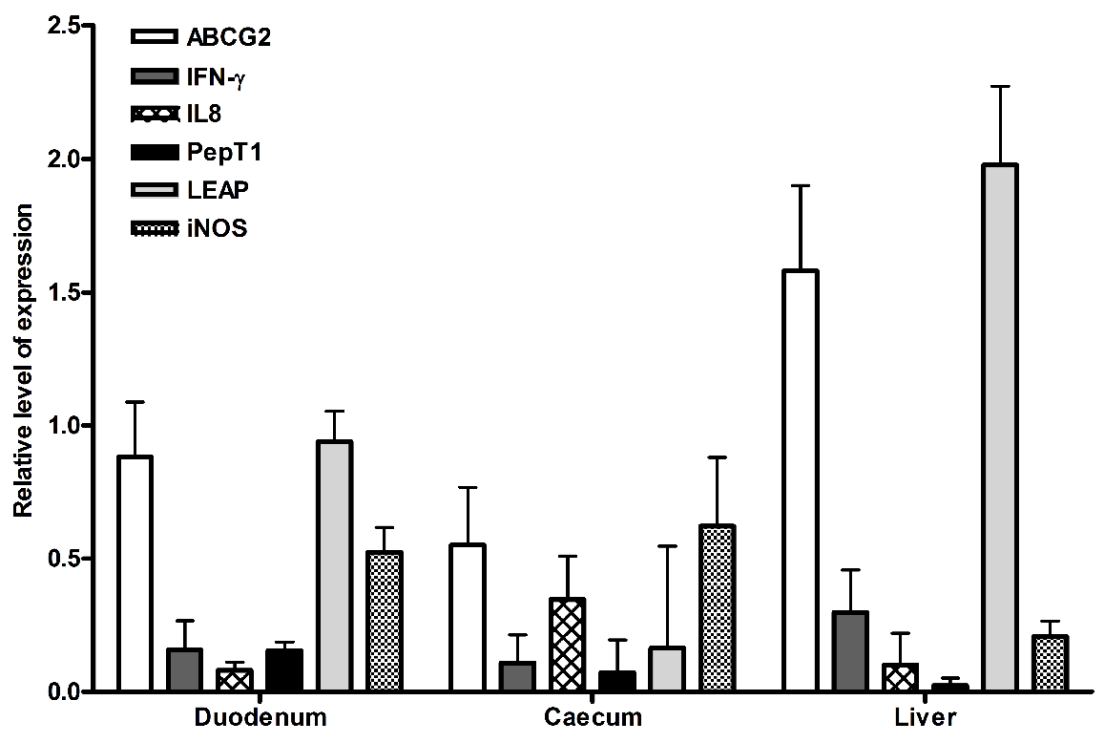

Fig. 1. Relative expression level of ABCG2, IFN- $\gamma$, IL8, PepT1, LEAP and iNOS mRNAs (mean and SD) in the duodenum, caecum and liver of healthy chickens $(\mathrm{n}=7)$.

technology was applied for the real-time PCR analysis by using $\mathrm{iQ}^{\mathrm{TM}}$ Sybr Green Supermix (Cat. No. 170-8885, Bio-Rad, Hercules, CA) and conducted according to the instructions of the manufacturer. The reaction was performed with an iCycler iQ PCR system (Bio-Rad, Hercules, CA) and analysed using MyiQ System Software, v. 1.0.410 (Bio-Rad Laboratories Inc.).

Each reaction went through a PCR cycle with a denaturation step at $95{ }^{\circ} \mathrm{C}$ for $20 \mathrm{~s}$, an annealing step specific for each set of primers for $30 \mathrm{~s}$ and an elongation step at $72{ }^{\circ} \mathrm{C}$ for $30 \mathrm{~s}$. After 35 cycles a melting curve was obtained by increasing the temperature with $0.5{ }^{\circ} \mathrm{C}$ every $10 \mathrm{~s}$ from $65{ }^{\circ} \mathrm{C}$ to $95{ }^{\circ} \mathrm{C}$ demonstrating the formation of only one product. Efficiencies for each reaction were estimated by Lin RegPCR 7.0 software. Beta-actin was used as an endogenous control. Gene expression data were presented using the algorithms outlined by Vandesompele et al. (2002).

\section{Statistical analysis and data presentation}

All data were analysed by Statistica 6.1 software (Statistica for Windows; StatSoft). Data are presented as median and range of minimum and maximum. They were checked for normality of the distribution and Mann-Whitney test was chosen for statistical evaluation. Differences between data in healthy and infected untreated chickens were considered significant at the level of $\mathrm{P}<0.05$.

\section{RESULTS}

Oral inoculation of chickens with $E$. tenella led to clear clinical signs, accompanied by high oocyst shedding and lesion scores six days after the challenge. The amount of oocysts for inoculation was cho- 


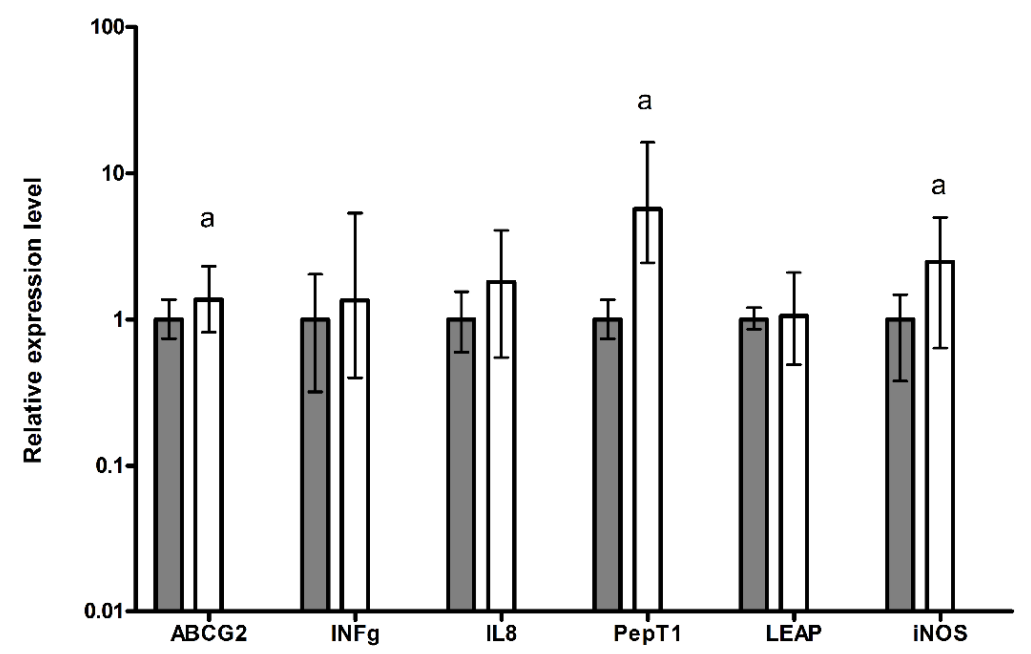

Fig. 2. Relative expression level of ABCG2, IFN $\gamma$, IL8, PepT1, LEAP and iNOS mRNAs (median and range of minimum and maximum) in the duodenum of healthy chickens $(\boldsymbol{\square}, \mathrm{n}=7)$ and infected with $E$. tenella animals 6 days after the challenge $(\square, n=7)$; a - statistically significant differences in comparison to healthy animals at $\mathrm{P}<0.05$.

sen to provoke a mild infection in order to have low mortality.

Factors from innate immune response: $L E A P, I F N-\gamma$, iNOS and $I L-8$

Relative expression levels of LEAP, IFN$\gamma$, iNOS and IL- 8 mRNAs in the duodenum, caecum and liver of healthy chickens are presented on Fig. 1. Analysis of the expression level of LEAP, IFN- $\gamma$ and IL-8 mRNAs showed no significant changes in the duodenum 6 days after the challenge with E. tenella. On the same day iNOS mRNA was significantly increased in the duodenum (Fig. 2).

IFN- $\gamma$ and iNOS mRNAs were significantly up-regulated in the caecum and the liver (Fig. 3 and 4). Apart from these genes, IL-8 mRNA expression in the liver was increased and LEAP mRNA decreased $(\mathrm{P}<0.05)$ after the infection. The data range between minimum and maximum was extremely high, probably due to the different degree of tissue damage in individual chickens.

Expression of transporter proteins PepT1 and $A B C G 2$

PepT1 and ABCG2 mRNA are constitutively expressed in the studied tissues. ABCG2 mRNA was found at the highest levels in the liver, followed by the duodenum and caecum (Fig. 1). On the sixth day after the challenge with $E$. tenella, ABCG 2 and PepT1 mRNAs were significantly increased in the duodenum, but remained unchanged in the caecum. ABCG2 mRNA was significantly up-regulated in the liver and in contrast, PepT1 mRNA was not affected.

\section{DISCUSSION}

Although E. tenella infection is strongly localised in the caecum and caecal tonsils it may contribute to the increased suscep- 


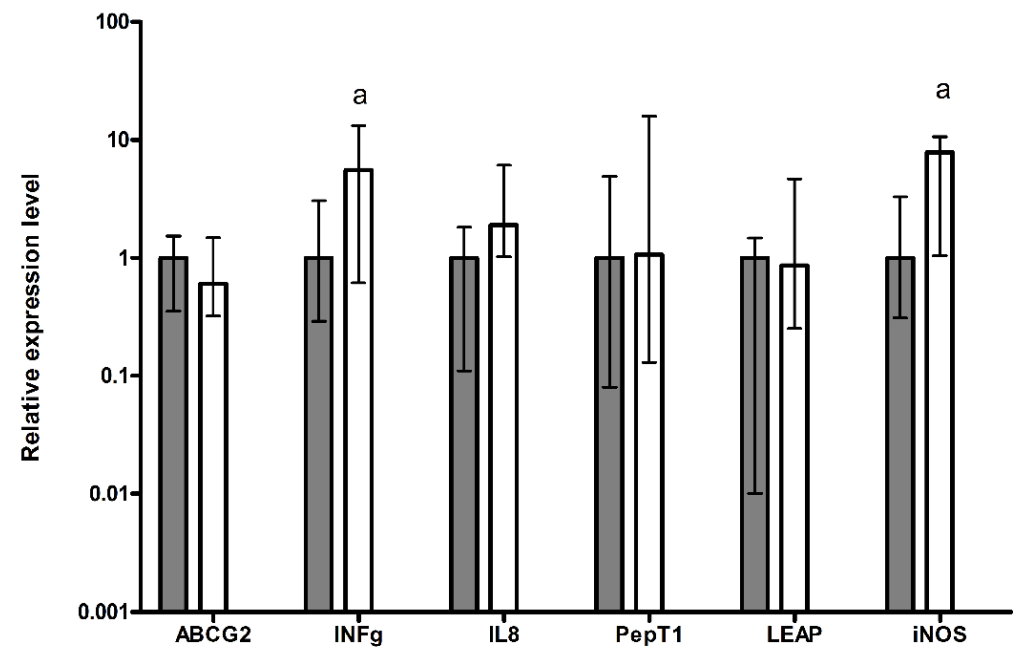

Fig. 3. Relative expression level of ABCG2, IFN $\gamma$, IL8, PepT1, LEAP and iNOS mRNAs (median and range of minimum and maximum) in the caecum of healthy chickens $(\boldsymbol{\square}, \mathrm{n}=7)$ and infected with E. tenella animals 6 days after the challenge $(\square, \mathrm{n}=7)$; a - statistically significant differences in comparison to healthy animals at $\mathrm{P}<0.05$.

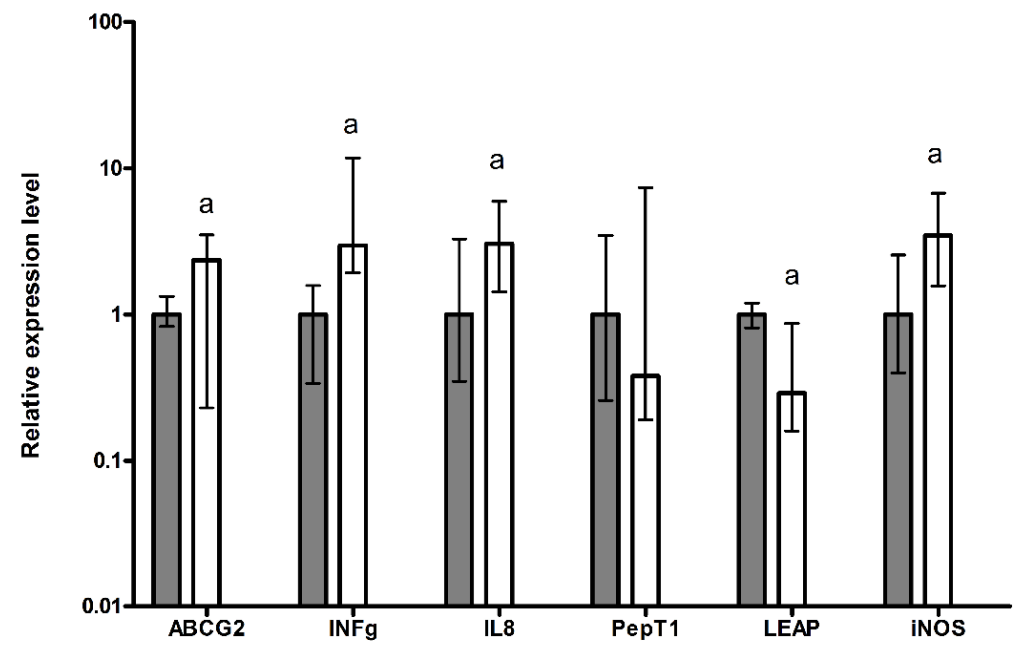

Fig. 4. Relative expression level of ABCG2, IFN $\gamma$, IL8, PepT1, LEAP and iNOS mRNAs (median and range of minimum and maximum) in the liver of healthy chickens $(\boldsymbol{\square}, \mathrm{n}=7)$ and infected with $E$. tenella animals 6 days after the challenge $(\square, \mathrm{n}=7)$; a - statistically significant differences in comparison to healthy animals at $\mathrm{P}<0.05$.

tibility of chickens to bacterial diseases often provoked by E. coli or Clostridium spp. (Yun et al., 2000). Therefore, in the present study, tissues from the caecum as a target for E. tenella, the duodenum and the liver were investigated. The initial part 
from the small intestine was chosen as a tissue unaffected by the coccidian invasion and the liver was included due to the generalised clinical signs and immune host response.

According to the results from our experiment, LEAP mRNA was expressed at a very low level in the caecum and only a tendency to down-regulation was observed in infected animals. The infection with E. tenella suppressed the response of the innate immunity by significant downregulation of the antimicrobial peptide levels in the liver, whereas no changes were observed in the duodenum at this time. These results are in line with the data reported by Sumners et al. (2011) and Casterlow et al. (2011), but these authors observed a significant depression of LEAP expression in the duodenum after a challenge with $E$. praecox. Downregulation of LEAP may result in an increased susceptibility of animals to bacterial infections, and hence may partly explain why chickens with clinical and subclinical coccidiosis are more sensitive to develop necrotic enteritis, associated with Clostridium spp. (Townes et al., 2009).

Among the cytokines, actively secreted by the Th1 lymphocytes during avian coccidiosis, IFN- $\gamma$ plays an important role (Yun et al., 2000). Similarly to the previously published results, IFN- $\gamma$ was significantly up-regulated in the caecum of infected animals if compared to uninfected controls (Yun et al., 2000; Laurent et al., 2001). These data, together with significantly increased IFN- $\gamma$ mRNA in the liver support the idea for its contribution to the protective host response against coccidian invasion. This induction of IFN- $\gamma$ may assist in the clearance of the infection and the development of adaptive immunity. As expected, due to the strong tissue affinity of E. tenella to the caecum, the mRNA expression level of this cytokine was not affected in the duodenum.

iNOS is considered as an important factor in inflammation. The production of iNOS is induced by IFN- $\gamma$ during $E$. tenella infection (Lillehoj \& Li, 2004). Higher levels of IFN- $\gamma$ during coccidiosis are positively correlated with the levels of iNOS mRNA and nitric oxide, and resistance of chickens to the disease (Laurent et al., 2001; Lillehoj \& Li, 2004). Taking into account these observations, significantly increased iNOS mRNA in the caecum and the liver can be related to the up-regulation of IFN- $\gamma$ mRNA in these tissues of infected animals. In caecal coccidiosis, iNOS mRNA induction is related to the haemorrhage that is frequently observed after E. tenella challenge (Laurent et al., 2001; Dalloul et al., 2007). Similar changes in iNOS mRNA were observed in the duodenum although IFN- $\gamma$ mRNA was not affected and this tissue was not a target for E. tenella.

In line with the results of Laurent et al. (2001), IL-8 mRNA was found to be constitutively expressed in the liver, caecum and duodenum in our experiments. According to the cited authors, IFN- $\gamma$ upregulates the synthesis of chemokines such as IL-8 after Eimeria spp. infection. Our data showed that E. tenella provoked a non-significant up-regulation of mRNA levels of this chemokine in the intestines as observed after E. tenella and E. maxi$m a$ infections in other experiments (Laurent et al., 2001; Hong et al., 2006; Cornelissen et al., 2009). The results from E. tenella infected chickens revealed significant up-regulation of IL-8 mRNA in the liver. The increase in IL- 8 is related to the activation of heterophils in chickens and our data indicate a systemic effect of IL-8 (Wigley \& Kaiser, 2003). Increased IL-8 is associated with an influx of 
heterophils in the epithelium and resulting damage by allowing the entry of coccidians (Cornelissen et al., 2009). The role of this cytokine is not well understood but it was assumed that its increase was related to the induction of IFN- $\gamma$ mRNA by recruitment of $\mathrm{Th} 1 \mathrm{CD}^{+}$, macrophages and monocytes. According to other investigations, IL-8 is expressed by various cells, such as epithelial and endothelial cells, and fibroblasts. By secretion of cytokines and chemokines these cells prolong the survival of Th1 lymphocytes (Karlson et al., 2007).

PepT1 mRNA was found at the highest levels in the duodenum, lower in the caecum and at the lowest levels in the liver from healthy chickens. Similar PepT1 expression in the intestines was observed by Gilbert et al. (2007) and it has been characterised as a transporter for peptides and peptidomimetic drugs. PepT1 mRNA was significantly up-regulated in the duodenum and was not changed in the other two tissues six days after the E. tenella challenge. This significant increase of PepT1 mRNA can be a result of the reduced feed intake during clinical coccidiosis and can be interpreted as a compensatory mechanism which allows for a better utilisation of peptide intake. Up-regulation of PepT1 mRNA in the duodenum was also observed in chickens under corticosterone-induced stress and in chickens under feed restriction (Hu et al., 2010; Madsen \& Wong, 2011).

ABCG2 and its protein (BCRP) belong to the family of $\mathrm{ABC}$ efflux transporters. Many drugs are substrates or modulators for this membrane transporter. Previous investigations showed that transepithelial permeability of sulfasalazine, a sulphonamide drug, can be decreased by the function of BCRP (Dahan \& Amidon, 2009; Jani et al., 2009). Therefore we included the measurement of the expression of this transporter in our experiments. In broiler chickens ABCG2 mRNA was found at highest levels in the liver, followed by the duodenum and the caecum as previously also observed in turkeys (Haritova et al., 2008). Six days after the challenge with E. tenella ABCG2 mRNA was significantly up-regulated in the duodenum and the liver which can be explained by its function in tissue defense against xenobiotics, including toxins (Leslie et al., 2005). The tendency to downregulation of this protein at mRNA level in the caecum after E. tenella infection is in line with the observed reduced expression of ABCG2 during active inflammation in ulcerative colitis (Englund et al., 2007).

In conclusion, E. tenella, considered as local coccidiosis in poultry chickens, can lead to alterations in the host immune response not only in the caecum but also in the liver and to the lesser degree in the duodenum. The results for ABCG2 and PepT1 mRNA indicate that significant changes can be expected in the integrity of small and large intestines.

\section{REFERENCES}

Casterlow, S., H. Li, E. R. Gilbert, R. A. Dalloul, A. P. McElroy, D. A. Emmerson \& E. A. Wong, 2011. An antimicrobial peptide is downregulated in the small intestine of Eimeria maxima-infected chickens. Poulry Science, 90, 1212-1219.

Cornelissen, J. B., W. J. Swinkels, W.A. Boersma \& J. M. Rebel, 2009. Host response to simultaneous infections with $E i$ meria acervulina, maxima and tenella: A cumulation of single responses. Veterinary Parasitology, 162, 58-66.

Dahan, A. \& G. L. Amidon, 2009. Small intestinal efflux mediated by MRP2 and BCRP shifts sulfasalazine intestinal permeability 
from high to low, enabling its colonic targeting. American Journal of Physiology. Gastrointestinal and Liver Physiology, 297, G371-377.

Dalloul, R. A. \& H. S. Lillehoj, 2006. Poultry coccidiosis: Recent advancements in control measures and vaccine development. Expert Review of Vaccines, 5, 143-163.

Dalloul, R. A., T. W. Bliss, Y. H. Hong, I. Ben-Chouikha, D. W. Park, C. L. Keeler \& H. S. Lillehoj, 2007. Unique responses of the avian macrophage to different species of Eimeria. Molecular Immunology, 44, 558-566.

Englund, G., A. Jacobson, F. Rorsman, P. Artursson, A. Kindmark. \& A. Rönnblom, 2007. Efflux transporters in ulcerative colitis: Decreased expression of BCRP (ABCG2) and Pgp (ABCB1). Inflammatory Bowel Diseases, 13, 291-297.

Gilbert, E. R., H. Li, D. A. Emmerson, K. E. Jr. Webb, \& E. A. Wong, 2007. Developmental regulation of nutrient transporter and enzyme mRNA abundance in the small intestine of broilers. Poultry Science, 86, 1739-1753.

Haritova, A., J. Schrickx, L. Lashev \& J. FinkGremmels, 2008. Expression of MDR1, MRP2 and BCRP mRNA in tissues of turkeys. Journal of Veterinary Pharmacology and Therapeutics, 31, 378-385.

Hong, Y. H., H. S. Lillehoj, S. H. Lee, R. A. Dalloul \& E. P. Lillehoj, 2006. Analysis of chicken cytokine and chemokine gene expression following Eimeria acervulina and Eimeria tenella infections. Veterinary Immunology and Immunopathology, 114, 209-223.

Hu, X. F., Y. M. Guo, B. Y. Huang, S. Bun, L. B. Zhang, J. H. Li, D. Liu, F. Y. Long, X. Yang \& P. Jiao, 2010. The effect of glucagon-like peptide 2 injection on performance, small intestinal morphology, and nutrient transporter expression of stressed broiler chickens. Poultry Science, 89, 1967-1974.

Jani, M., P. Szabó, E. Kis, E. Molnár, H. Glavinas \& P. Krajcsi, 2009. Kinetic characterization of sulfasalazine transport by human ATP-binding cassette G2. Biological \& Pharmaceutical Bulletin, 32, 497-499.

Karlson, T., L. De, C. V. Whiting \& P. W. Bland, 2007. Proinflammatory cytokine synthesis by mucosal fibroblasts from mouse colitis is enhanced by interferon- $\gamma$ mediated up-regulation of CD40 signalling. Clinical and Experimental Immunology, 147, 313-323.

Laurent, F., R. Mancassola, S. Lacroix, R. Menezes \& M. Naciri, 2001. Analysis of chicken mucosal immune response to Eimeria tenella and Eimeria maxima infection by quantitative reverse transcription-PCR. Infection and Immunity, 69, $2527-2534$

Leslie, E. M., R. G. Deeley \& S. P. Cole, 2005. Multidrug resistance proteins: Role of P-glycoprotein, MRP1, MRP2, and BCRP (ABCG2) in tissue defense. Toxicology and Applied Pharmacology, 204, 216-237.

Lillehoj, H. S. \& G. Li, 2004. Nitric oxide production by macrophages stimulated with Coccidia sporozoites, lipopolysaccharide, or interferon-gamma, and its dynamic changes in SC and TK strains of chickens infected with Eimeria tenella. Avian Diseases, 48, 244-253.

Madsen, S. L. \& E. A. Wong, 2011. Expression of the chicken peptide transporter 1 and the peroxisome proliferator-activated receptor $\alpha$ following feed restriction and subsequent refeeding. Poultry Science, 90, 2295-2300.

Michailidis, G., 2010. Expression of chicken LEAP-2 in the reproductive organs and embryos and in response to Salmonella enterica infection. Veterinary Research Communications, 34, 459-471.

Rubio-Aliaga, I. \& H. Daniel, 2008. Peptide transporters and their roles in physiological processes and drug disposition. Xenobiotica, 38, 1022-1042.

Sumners, L. H., K. B. Miska, M. C. Jenkins, R. H. Fetterer, C. M. Cox, S. Kim \& R. A. 
Influence of experimentally induced Eimeria tenella infection on gene expression of some host ...

Dalloul, 2011. Expression of Toll-like receptors and antimicrobial peptides during Eimeria praecox infection in chickens. Experimental Parasitology, 127, 714-718.

Townes, C. L., G. Michailidis, \& J. Hall, 2009. The interaction of the antimicrobial peptide cLEAP-2 and the bacterial membrane. Biochemical and Biophysical Research Communications, 387, 500-503.

van der Heijden, J., M. C. de Jong, B. A. Dijkmans, W. F. Lems, R. Oerlemans, I. Kathmann, C. G. Schalkwijk, G. L. Scheffer, R. J. Scheper \& G. Jansen, 2004. Development of sulfasalazine resistance in human $T$ cells induces expression of the multidrug resistance transporter ABCG2 (BCRP) and augmented production of TNF alpha. Annals of the Rheumatic Diseases, 63, 138-143.

Vandesompele, J., K. De Peter, F. Pattyn, B. Poppe, N. Van Roy, A. De Paepe \& F. Speleman, 2002. Accurate normalization of real-time quantitative RT-PCR data by geometric averaging of multiple internal control genes. Genome Biology, 3, research0034-research0034.11

Wigley, P. \& P. Kaiser, 2003. Avian cytokines in health and disease. Revista Brasileira de Ciência Avicola, 5, 1-14.

Yun, C. H., H. S. Lillehoj \& K. D. Choi, 2000. Eimeria tenella infection induces local gamma interferon production and intesti- nal lymphocyte subpopulation changes. Infection and Immunity, 68, 1282-1288.

Zhang, L., R. Liu, L. Ma, Y. Wang, B. Pan, J. Cai \& M. Wang, 2012. Eimeria tenella: expression profiling of toll-like receptors and associated cytokines in the cecum of infected day-old and three-week old SPF chickens. Experimental Parasitology, 130, 442-448.

Paper received 20.01.2015; accepted for publication 13.03.2015

\section{Correspondence:}

Prof. Anelia Milanova

Department of Pharmacology, Physiology of Animals and Physiological Chemistry,

Faculty of Veterinary Medicine,

Trakia University,

Students' Campus,

6000 Stara Zagora, Bulgaria

Phone: 0035942699696

e-mail: akmilanova@gmail.com 